\title{
Fiber Bragg grating as a UVA sensor
}

\author{
P. Lesiak ${ }^{1}$, A. Widomski ${ }^{1}$, Ł. Szelągowski ${ }^{1}$, P. Sobotka $^{1}$, A. Dużyńska ${ }^{1}$, A. Wróblewska ${ }^{1}$, K. Markowski $^{2}$, \\ T. Osuch, ${ }^{2,3}$ and T.R. Woliński ${ }^{1}$, \\ ${ }^{1}$ Faculty of Physics, Warsaw University of Technology, Koszykowa 75, 00-662 Warszawa, \\ ${ }^{2}$ Faculty of Electronics and Information Technology, Warsaw University of Technology, Nowowiejska 15/19, \\ 00-665 Warszawa \\ ${ }^{3}$ National Institute of Telecommunications, Szachowa 1, 04-894 Warszawa
}

Received March 28, 2018; accepted March 30, 2018; published March 31, 2018

\begin{abstract}
The idea of this paper implies the possibility to exploit the properties of graphene oxide (GO) to design a fiber Bragg grating (FBG) UVA radiation sensor. The idea assumes that a temperature change around the fiber can be induced by UVA radiation. UVA lighting will increase the internal energy of the GO and consequently locally raise the temperature on the surface of the optical fiber with an FBG sensor, changing the Bragg wavelength.
\end{abstract}

The synthesis and disintegration of molecules through the processes of bond activation or deactivation are caused by the strong interaction of ultraviolet radiation with chemical compounds [1]. A common means of epoxy resin production techniques is the formation of complex molecules due to the activation of bonds, whose structure depends on molecular weight. By controlling this parameter it is possible to obtain liquids or plastic solids. The final product is obtained by spatial crosslinking with hardeners that can be activated with UVA radiation. The properties of the product (such as mechanical strength or chemical resistance) strongly depend on curing conditions and degree of cross-linking [2]. This implies the need to create a non-invasive method for controlling the above mentioned process [3]. One of the ways to realize this is by the use of a fiber Bragg grating.

Fiber optic sensors have many advantages over conventional sensors [4], such as small size, electrical safety or immunity to electromagnetic interference. The immunity of sensors to intense electromagnetic fields, for example near a UV lamp [5], is especially desirable, which makes fiber optics sensors ideal for using in such conditions.

Graphene oxide (GO) attracts attention in the field of UVA radiation detection due to its high UVA absorption [6]. Typically, an aqueous solution of graphene oxide shows an absorbance maximum at $230 \mathrm{~nm}$ in its absorbance spectrum, while other wavelengths (visible and infrared) are not absorbed to a significant extent. The internal energy of GO increases under UV illumination and consequently its surface temperature rises. This can be used to affect, in many ways, light propagation in fibers covered by GO [7].
In this paper we investigate the possible use of graphene oxide to measure UV radiation with a fiber Bragg grating sensor (FBG). Fiber Bragg gratings can be used for direct measurements, have good linearity, wavelength multiplexing capacity, high resistance in harsh environments [8-9].

A typical FBG has a sinusoidal refractive index variation over a defined length [11-12]. The reflected wavelength $\left(\lambda_{B}\right)$, called the Bragg wavelength, is defined by the relationship:

$$
\lambda_{B}=2 n_{\text {eff }} \Lambda,
$$

A change in the temperature in the immediate vicinity of the FBG changes the grating period (due to thermal expansion) and the effective refractive index in the optical fiber in which the grating is written (due to the thermooptic effect). As a result, the Bragg wavelength shifts. The influence of temperature changes on an FBG resonance wavelength is described by the following formula [3]:

$$
\Delta \lambda_{B}=2\left(\Lambda \frac{\partial n_{e f f}}{\partial T}+n_{\text {eff }} \frac{\partial \Lambda}{\partial T}\right) \Delta T .
$$

Typical temperature sensitivity for Bragg wavelength $1550 \mathrm{~nm}$ is equal to $10 \mathrm{pm} / \mathrm{K}$.

Graphene oxide films were fabricated via a vacuum filtration method [4] and deposited on optical fibers. In detail, GO thin films were produced using the water suspension of GO from Graphenea. The starting suspension with a concentration of $4 \mathrm{mg} / \mathrm{ml}$ was diluted by deionized water to achieve a concentration of $0.1 \mathrm{mg} / \mathrm{ml}$. Next, the determined volume of suspension was vacuum filtered onto a mixed cellulose ester membrane (MCE, $0.45 \mu \mathrm{m}$ pore-size, $25 \mathrm{~mm}$ diameter) to form a GO thin film. Additionally, the GO film was left under air flow in a filtration setup to complete drying. Then, the dry GO film on the MCE filter was cut to prepare $2 \times 3 \mathrm{~mm}^{2}$ pieces, which were immediately immersed in an acetone bath to dissolve the filter. When GO films were clean and free from any residual membrane, the acetone was replaced by an ethanol/water solution with the ratio of 
1:1. Finally, each of the pure GO thin films was transferred from the alcohol solution on a selected optical fiber and gently dried with a nitrogen stream (Fig. 1). The thickness of the GO film was measured by atomic force microscopy (AFM) using a flat substrate and equals $100 \mathrm{~nm}$.

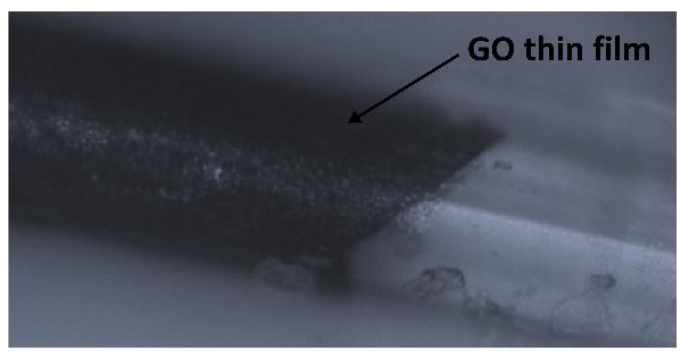

Fig. 1. The image of optical fiber partially covered by a film of graphene oxide observed in an optical microscope (magnification $\times 20$ ).

An example Raman spectrum of graphene oxide film covering the optical fiber is presented in Fig. 2 (the peak positions and their intensity values were obtained through a Lorentz fit of the Raman spectra). We observed two main bands: D $\left(1345.9 \mathrm{~cm}^{-1}\right)$ and $\mathrm{G}\left(1590.5 \mathrm{~cm}^{-1}\right)$, which are characteristic of a graphene oxide material [5]. The relative intensity ratio of these modes $\left(\mathrm{I}_{\mathrm{D}} / \mathrm{I}_{\mathrm{G}}=1.36\right)$ confirms the presence of the GO film on the optical fiber [6]. Raman spectra were collected using a 514-laser line in a Renishaw inVia spectrometer in ambient conditions. Measurements were performed for low laser power (below $1 \mathrm{~mW}$ ) to avoid sample heating. Importantly, the spectra were reproducible all over the sample.

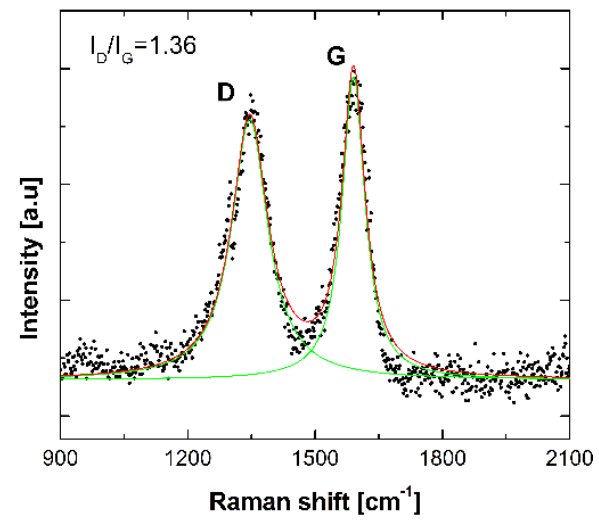

Fig. 2. Raman spectrum of a GO thin film deposited on optical fiber. Peak positions and intensities were obtained from Lorentzian fit.

To perform the experiment a beam of light with a spectral width of $100 \mathrm{~nm}$ and a central wavelength of $1550 \mathrm{~nm}$ was inserted into the optical fiber. The Bragg wavelength returned to the circulator and left it through the third exit, which led to an optical spectrum analyzer. The GO cover was applied to the surface of the fiber in the place where the FBG was inscribed. Two laser beams with wavelengths of $\lambda=325 \mathrm{~nm}$ and $\lambda=543 \mathrm{~nm}$ were focused on the covered parts of the fiber (Fig. 3). The heated part of the fiber was the same length as the FBG inscribed in the fiber, which was $4 \mathrm{~mm}$.

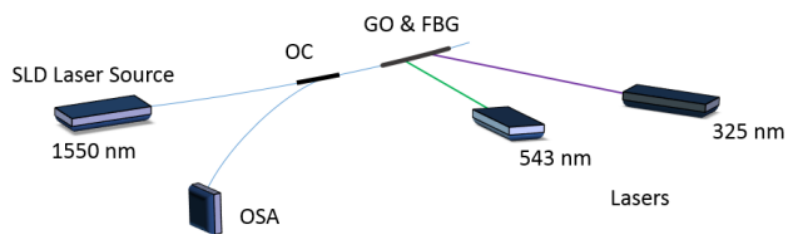

Fig. 3. Measurement setup, where OC is optical circulator and OSA is optical spectrum analyzer.

The results presented in Fig. 4 and Fig. 5 show that the UVA light absorbed by GO warms up the FBGs. The temperature changes on the FBGs was clearly observed in our experiment results. Additionally, the change in the Bragg wavelength is characterized by linear dependence on UVA radiation (Fig. 6).

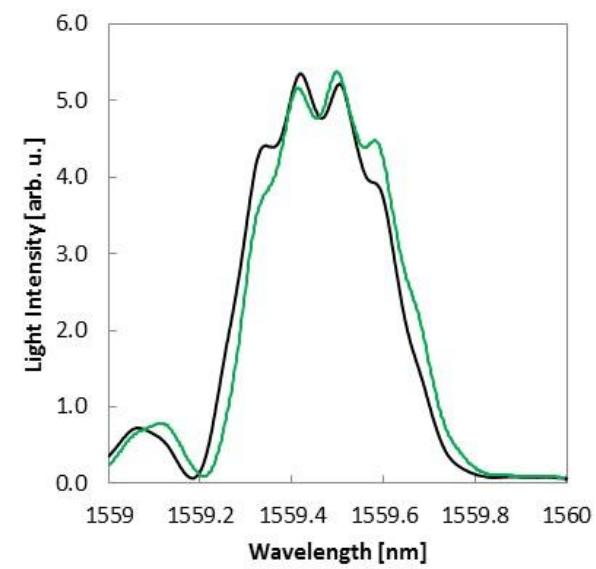

Fig. 4. Bragg wavelength shift under $543 \mathrm{~nm}$ laser light (1mW) focused on GO. Laser off (black) and laser on (green).

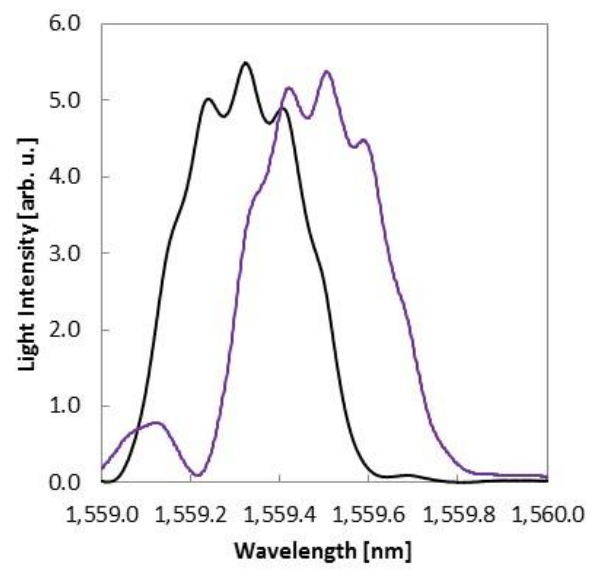

Fig. 5. Bragg wavelength shift under $325 \mathrm{~nm}$ laser light $(2 \mathrm{~mW})$ focused on GO. Laser off (black) and laser on (violet). 


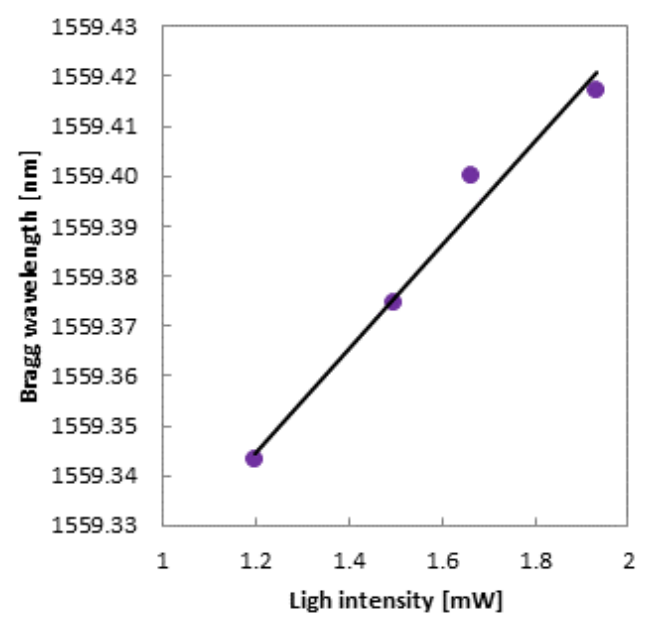

Fig. 6. Bragg wavelength in the function of $325 \mathrm{~nm}$ laser light intensity focused on $\mathrm{GO}$.

For the green laser, the light intensity was $1 \mathrm{~mW}$ and for a laser with a wavelength of $325 \mathrm{~nm}-2 \mathrm{~mW}$. In the case of the UVA source, the power was limited so as not to overheat GO.

For green light, the observed Bragg wavelength shift was $16.5 \mathrm{pm}$, corresponding to FBG sensor heating by $1.5 \mathrm{~K}$. For the light source $325 \mathrm{~nm}$, the Bragg wavelength shift was $166 \mathrm{pm}$, which corresponds to the heating of the FBG sensor by $16.6 \mathrm{~K}$. The calculated sensitivity to green and UVA laser sources was $16.5 \mathrm{pm} / \mathrm{K}$ and $83.0 \mathrm{pm} / \mathrm{K}$, respectively. The sensitivity of the presented solution strongly depends on the positioning of the FBG and GO layer in relation to each other. Additionally, the UVA sensor requires uniform illumination of the GO layer. Non uniform illumination creates a temperature gradient on the FBG that can change the shape of the reflected spectrum and makes the signal unreadable.

In this paper a possible use of the graphene oxide to measure UV radiation by using an FBG was investigated. The obtained results show that UV light focused on the FBG covered by GO induces Bragg wavelength changes that are observed as a spectral shifts in measured reflected spectrum. However, the proposed solution requires further optimization work.

\section{References}

[1] Z.N. Azwa et al., Mat. Design 47, 424 (2013).

[2] B. Rånby, Polymer Ing. Science 38(8), 1229 (1998).

[3] P. Lesiak, et al., Measur.: J. Intern. Measur. Confed. 45(9), 2275 (2012).

[4] C. Fitzpatrick et al., Meas. Sci. Technol. 14(8), 1477 (2003).

[5] A.V. Joža et al., Telfor. J. 4(2), 133 (2012).

[6] P. Lesiak, et al., Phot. Lett. Poland 7(4), 124 (2015).

[7] B. Qi et al., IOME Austr. Mat. Forum 27, 93 (2004).

[8] E. Chehura et al., Smart Mat. Struct. 13, 888 (2004).

[9] K. Schroeder et al., Measur. Science Techn. 17, 1167 (2006).

[10] Z. Zhou et al., Measur. 44(9), 1499 (2011).

[11] Z.C. Wu et al., Science 305, 1273 (2004).

[12] A. Jorio, M. Dresselhaus, R. Saito, G.F. Dresselhaus, Raman Spectroscopy in Graphene Related Systems (Wiley-VCH, 2011).

[13] G. Sobon, et al., Opt. Express 20, 19463 (2012).

[14] G. Eda et al., Nature Nanotechnology 3, 270 (2008).

[15] H.J. Kim et al., Scientific Reports 4, 5176 (2014).

[16] A. Wróblewska et al., J. Physics: Condensed Matt. 29, 475201 (2017). 